\title{
50 ans Le laser aux extrémités du spectre : LASER le laser térahertz
}

Aux frontières de l'électronique et de l'optique, les lasers térahertz ont été inaccessibles dans une configuration utilisable pendant longtemps. Mais les innombrables applications de ces ondes électromagnétiques ont encouragé les chercheurs à relever le défi et ces derniers sont en passe de réussir à obtenir une technologie fiable, notamment grâce aux lasers à cascade quantique.

\section{Carlo SIRTORI}

Université Paris Diderot

carlo.sirtori@univ-paris-diderot.fr sion spontanée devient tellement inefficace que l'effet d'amplification par émission stimulée ne se déclenche plus automatiquement (à ces basses fréquences, on parle d'ailleurs plutôt de maser).

\section{Le térahertz: domaine de l'optique ou de l'électronique?}

Dans l'esprit des scientifiques persiste une séparation nette entre l'électronique et l'optique. En particulier, quand on fait référence à la technologie des semi-conducteurs, la distinction est claire avec, d'une part, les circuits intégrés à base de transistors capable de produire des ondes électromagnétiques de dizaines de gigahertz $\left(1 \mathrm{GHz}=10^{9}\right.$ cycles/ seconde) et, d'autre part, les diodes laser et la technologie des télécoms qui produisent de la lumière aux fréquences de plusieurs centaines de térahertz ( $\left.1 \mathrm{THz}=10^{12} \mathrm{cycles} / \mathrm{seconde}\right)$. L'énorme écart spectral, la nature distincte de la physique de leurs dispositifs constituants et des raisons commerciales ont éloigné ces deux branches de la technologie que les équations de Maxwell ne permettent pourtant pas de distinguer. La discontinuité abrupte qui sépare ces deux mondes dans les esprits est, au contraire, une transition très douce qui s'étale dans une large plage spectrale où les concepts de l'électronique et de l'optique se mélangent. Il est intéressant de noter que la gamme spectrale des térahertz est la dernière dans laquelle l'effet laser peut être observé : si l'on réduit encore l'énergie des photons et que l'on s'approche de la région des ondes millimétriques, l'émis-

\section{Un domaine spectral peu exploité...}

Encore aujourd'hui, la région térahertz du spectre électromagnétique reste largement inexploitée tant il est difficile de réaliser des sources de rayonnement cohérent efficaces. Il est presque impossible d'obtenir des courants oscillants à ces hautes fréquences dans les circuits intégrés car les capacités et les résistances des circuits freinent les électrons et empêchent la formation d'une macro-polarisation capable de produire des ondes électromagnétiques aux fréquences térahertz.

C'est donc ici que les lasers deviennent extrêmement intéressants car ils permettent la génération de lumière par le biais de l'émission stimulée, à partir d'une polarisation à l'échelle atomique qui est - en principe - sans dissipation. Cependant, comme l'énergie des photons térahertz est très faible (entre 100 et 1000 fois plus petite que celle du visible), il faut obtenir l'inversion de population entre deux niveaux d'énergie qui sont séparés d'environ $10 \mathrm{meV}$, c'est-àdire bien moins que l'énergie d'agitation thermique à température ambiante ( $25 \mathrm{meV}$ ).

Peupler l'état excité de la transition laser en laissant quasiment vide le niveau fondamental à ces longueurs d'onde est donc très difficile. La faisabilité en a cependant été démontrée dans des systèmes à base de gaz moléculaires dans les années soixante-dix et dans des cristaux macroscopique de germanium dans les années quatre-vingt. Ces systèmes fonctionnent néanmoins dans des conditions peu pratiques : les lasers à gaz ont besoin de cavités optiques de quelques mètres et les lasers germanium doivent être dans un champ magnétique intense et à température inférieure à $-240^{\circ} \mathrm{C}$ ! Cela a fait que l'impact technologique de ces lasers est toujours resté extrêmement limité.

\section{.. qui se découvre des applications innombrables...}

Cependant, au début des années 2000, les scientifiques ont commencé à mettre en évidence les propriétés uniques des ondes térahertz : en plus de correspondre aux transitions d'un grand nombre de molécules, elles sont facilement transmises par toute une série de substances non métalliques (dont les matériaux plastiques et certains types de tissus), contrairement aux longueurs d'onde optiques. Ce sont donc des innombrables domaines d'exploitation potentielle (de l'imagerie dans le domaine de la sécurité à la recherche pharmaceutique en passant par le test non destructif, l'imagerie et l'analyse du tissu humain, la radioastronomie et la surveillance de l'atmosphère...) qui ont encouragé une recherche sur les dispositifs semi-conducteurs pour obtenir des sources compactes et efficaces. 
puis des technologies pertinentes

Et, dans cette quête de la source idéale, les lasers à cascade quantique (ou $\mathrm{QCL}$ pour Quantum Cascade Lasers) portent tous les espoirs. Dans ces lasers à semiconducteurs, le gain se développe entre deux niveaux d'énergie électronique dans un puits quantique de la bande de conduction. À la différence des diodes laser, l'émission stimulée des photons ne se produit donc pas à l'énergie du gap du semi-conducteur, mais elle peut être variée simplement en changeant la taille des puits quantiques (et donc la séparation entre les niveaux d'énergie).

Ces lasers, démontrés pour la première fois aux Bell Labs (USA) en 1994, ont tout d'abord

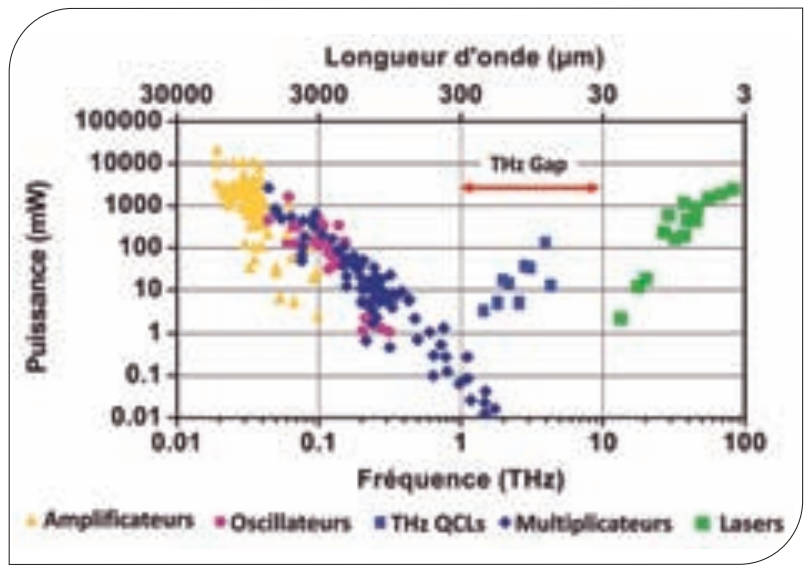
été réalisés dans le moyen infrarouge $(\lambda=5-10 \mu \mathrm{m})$, donc à quelques dizaines de térahertz.

Pendant plusieurs années, les chercheurs ont essayé d'atteindre la région spectrale du térahertz, mais ils se sont heurtés au problème du guidage de la lumière à grandes longueurs $d$ 'onde $(\lambda=100-300 \mu \mathrm{m})$ par des structures diélectriques (ce qui est la technologie adoptée pour les diodes laser).

Ce problème a finalement été résolu lorsque l'approche d'un guide diélectrique a été abandonnée au profit de guide d'onde typique de I'hyperfréquence, où le confinement du rayonnement électromagnétique est obtenu par des couches métalliques.

Et c'est ainsi que la «course au premier laser semi-conducteur térahertz " a été gagnée en 2002 par une collaboration italo-britannique pilotée par une équipe de l'Ecole Normale de Pise, qui a réalisé un laser à cascade quantique fonctionnant à $4 \mathrm{THz}$, en mêlant les domaines de l'optique et de l'hyperfréquence avec, d'une part, le laser et, d'autre part, la ligne de transmission microstrip (ou micro-ruban).

\section{Aller plus loin.。}

Malgré ce succès notoire qui a permis de réaliser des lasers térahertz à des puissances intéressantes (figure 1) pour un encombrement très réduit (figure 2 ), le fait que ces lasers à cascade quantique soient aujourd'hui encore limités à de très basses températures (de l'ordre de $-100^{\circ} \mathrm{C}$ ) est un inconvénient majeur pour le développement d'une technologie basée sur ces dispositifs.

Puissance de rayonnement électromagnétique aux alentours de $1 \mathrm{THz}$ pour les sources à l'état solide. II est à noter que les données des $\mathrm{THz} \mathrm{QCLs}$ sont prises à température cryogénique.

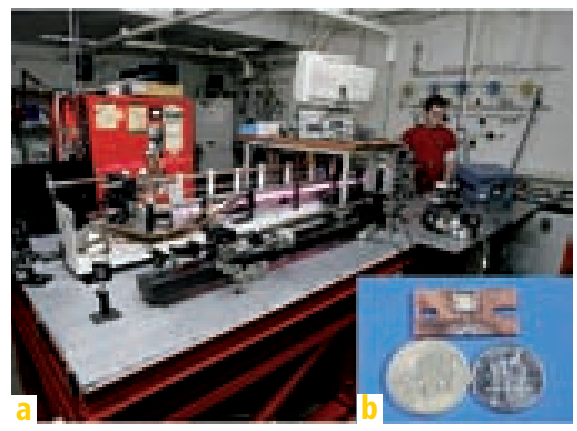

Figure 2. Illustration des tailles typiques d'un laser térahertz à gaz (a) et d'un laser térahertz à cascade quantique (b).

Cependant, on peut se poser la question fondamentale " $y$ a-t-il une vraie limitation physique qui empêche le fonctionnement de ces lasers à température ambiante ?»... puis y répondre " honnêtement, non !». Et cette recherche d'une solution à température ambiante - loin d'être évidente - est un problème idéal pour les chercheurs des dispositifs quantiques!

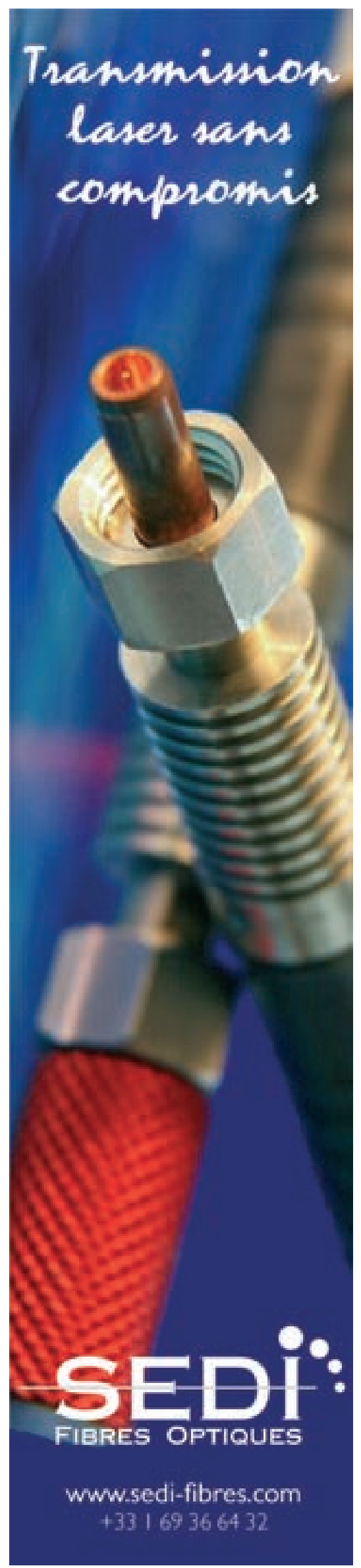

